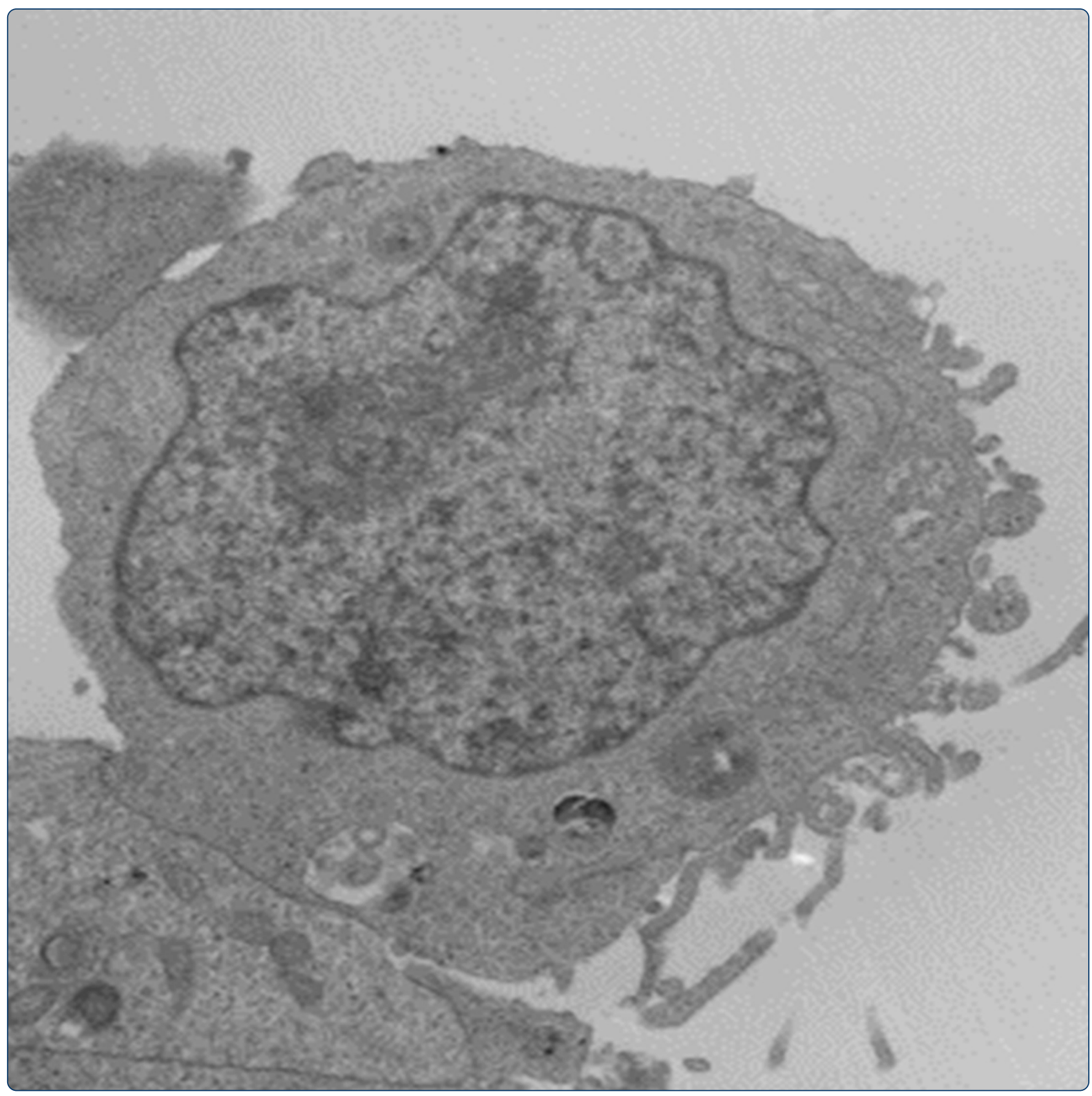

Combination treatment with ABT-737 and chloroquine in preclinical models of small cell lung cancer

Zinn et al. 


\title{
Combination treatment with ABT-737 and chloroquine in preclinical models of small cell lung cancer
}

Rebekah L Zinn, Eric E Gardner, Irina Dobromilskaya, Sara Murphy, Luigi Marchionni, Christine L Hann and Charles M Rudin*

\begin{abstract}
Background: New therapies are urgently needed for patients with small cell lung cancer (SCLC). Chemotherapy and targeted therapies, including the BCl-2 inhibitor ABT-737, may induce tumor cell autophagy. Autophagy can promote survival of cancer cells under stress and comprise a pathway of escape from cytotoxic therapies.

Methods: We explored the combination of ABT-737 and chloroquine, an inhibitor of autophagy, in preclinical models of SCLC. These included cell culture analyses of viability and of autophagic and apoptotic pathway induction, as well as in vivo analyses of efficacy in multiple xenograft models.

Results: Combination treatment of SCLC lines with ABT-737 and chloroquine decreased viability and increased caspase-3 activation over treatment with either single agent. ABT-737 induced several hallmarks of autophagy. However, knockdown of beclin-1, a key regulator of entry into autophagy, diminished the efficacy of ABT-737, suggesting either that the effects of chloroquine were nonspecific or that induction but not completion of autophagy is necessary for the combined effect of ABT-737 and chloroquine. ABT-737 and chloroquine in SCLC cell lines downregulated $\mathrm{MCl}-1$ and upregulated NOXA, both of which may promote apoptosis. Treatment of tumorbearing mice demonstrated that chloroquine could enhance ABT-737-mediated tumor growth inhibition against $\mathrm{NCl}-\mathrm{H} 209$ xenografts, but did not alter ABT-737 response in three primary patient-derived xenograft models.

Conclusion: These data suggest that although ABT-737 can induce autophagy in SCLC, autophagic inhibition by choroquine does not markedly alter in vivo response to ABT-737 in relevant preclinical models, arguing against this as a treatment strategy for SCLC.
\end{abstract}

Keywords: Autophagy, Apoptosis, ABT-737, Chloroquine, Primary xenograft

\section{Background}

Autophagy is an evolutionarily conserved, reversible process in which the cell degrades its own cytoplasmic components utilizing lysosomal pathways. Typically, autophagy is activated under conditions of cell stress, including nutrient deprivation, environmental stressors, accumulation of misfolded proteins, and growth factor signal interruption. Cytoplasmic components are sequestered into double-membraned vesicles, or autophagosomes, which then fuse with lysosomes and the contained material

\footnotetext{
* Correspondence: rudin@jhmi.edu

Department of Oncology, The Sidney Kimmel Comprehensive Cancer Center at the Johns Hopkins University School of Medicine, Cancer Research Building 2, Room 5441550 Orleans Street, Baltimore, MD, USA
}

is degraded to amino acids, nucleotides, sugars, and fatty acids that are released into the cytoplasm for biosynthesis and metabolism [1,2].

Recent evidence suggests that autophagy is frequently activated in tumor cells treated with chemotherapy and radiation [3-7]. While persistent activation of autophagy may lead to tumor cell death, accumulating evidence suggests that it can serve as a protective pathway for cells to escape apoptotic cell death, allowing survival through periods of stress, growth factor withdrawal, or nutrient deprivation $[8,9]$. Several studies have shown that autophagy delayed apoptotic death in cancer cells undergoing chemotherapeutic treatment. Treatment of these cells with inhibitors of autophagy, such as chloroquine, or knockdown of essential

\section{Biomed Central}


autophagy genes (beclin-1, ATG genes) resulted in enhanced therapy-induced apoptosis [10-14]. Chloroquine acts as a lysosomal $\mathrm{pH}$ inhibitor, therefore preventing the fusion of autophagosomes with lysosomes and inhibiting late stages of autophagy $[15,16]$. These findings have led to the initiation of multiple clinical trials combining autophagy inhibitors and chemotherapeutic agents for diverse cancer types [17].

Small cell lung cancer (SCLC) comprises approximately $15 \%$ of all lung cancers and is a remarkably aggressive disease [18]. SCLC is characterized by rapid proliferation and early dissemination to metastatic sites. While standard of care platinum-based chemotherapy induces responses in up to $80 \%$ of newly diagnosed SCLC cases, these responses are short-lived: the median survival in patients with advanced SCLC is approximately 9 months from the time of diagnosis [19]. New therapies are critically needed for this disease.

The anti-apoptotic protein Bcl-2 is overexpressed in the majority of SCLC cases [20,21]. Suppression of Bcl-2 can trigger apoptosis in SCLC, and enhance the anti-cancer efficacy of cytotoxic therapies [22]. ABT-737, a Bcl-2 homology domain 3 mimetic (BH3), which inhibits the activity of $\mathrm{Bcl}-2$ and $\mathrm{Bcl}-\mathrm{X}_{\mathrm{L}}$, has been shown to have potent activity against many SCLC cell lines [23]. However, the efficacy of ABT-737 is variable across SCLC cell lines, and this agent has only modest single agent activity against most primary xenograft models of SCLC [24]. Consistent with the observed level of activity in primary xenografts, while clinical trials of ABT-263 (an orally bioavailable derivative of ABT-737 for human use) have shown pharmacodynamic evidence of on-target activity, the overall response rate in SCLC patients has been disappointing $[25,26]$.

We hypothesized that SCLC resistance to ABT-737 may be in part due to cancer cell induction of autophagy. Bcl-2 has been shown to directly bind and inhibit Beclin-1 (BECN1), a critical mediator of initiation of autophagy [27]. Treatment of HeLa and MCF7 cells with ABT-737 or gossypol, another purported BH3 mimetic, has been shown to inhibit the interaction of Beclin-1 and $\mathrm{Bcl}-2 / \mathrm{Bcl}-\mathrm{X}_{\mathrm{L}}$ and stimulate autophagy [28-30]. We sought to define whether the treatment of SCLC cell lines with ABT-737 induces autophagy and whether this induction is a mechanism of therapeutic resistance. We also assessed the in vivo efficacy of the combination of ABT-737 and chloroquine using patient-derived xenograft (PDX) models of SCLC.

\section{Results}

To assess whether an inhibitor of autophagy could affect viability of SCLC cell lines, and could augment the efficacy of the Bcl-2 inhibitor ABT-737, we initially treated several SCLC cell lines (H82, H209, and H345) with
ABT-737, chloroquine, or the combination of both agents. Quantitative assessment of viable cells was performed at 72 hours using a standard MTS assay. In all three cell lines, the combination of ABT-737 with chloroquine significantly decreased cell viability relative to either agent alone ( $\mathrm{p}<0.002$ for all three, see Figure $1 \mathrm{~A}$ ).

We have previously reported on a series of PDX tumors, generated by direct transfer of human tumor into immunosuppressed mice, which we have used as a preclinical platform for testing novel therapeutic strategies, including ABT-737 [24,31]. For correlative in vitro analyses, we have also generated derivative cell lines from two of these primary xenografts, LX22 and LX33. Cell lines derived from LX22 and LX33 both demonstrated greater loss of viability, as determined by MTS, in response to the combination of ABT-737 and chloroquine than to either agent alone ( $\mathrm{p}<0.003$ for both, see Figure $1 \mathrm{~B})$.

These results suggested that inhibition of autophagy could enhance the efficacy of ABT-737. Alternatively, chloroquine could be having a cytotoxic effect unrelated to inhibition of autophagy in these cells. We interrogated this intersection between apoptotic and autophagic pathways using an alternative pharmacologic inhibitor of autophagy, 3-methyladenine (3MA). Treatment with 3MA, with or without ABT-737, in H209 and H345 cells resulted in similar results to those obtained with chloroquine: clear evidence of enhanced cytotoxic response in cells treated with the combination relative to either agent alone ( $\mathrm{p}<0.0015$ for both, see Figure $1 \mathrm{C}$ ).

We next sought to characterize whether the combinatorial effects observed in cells treated with ABT-737 and chloroquine were associated with enhanced apoptosis, as hypothesized. Caspase-3 activation is a central hallmark of apoptotic induction through both the extrinsic and intrinsic apoptotic pathways. The combination of ABT-737 and chloroquine resulted in higher levels of caspase-3 activation relative to either treatment alone in several SCLC lines (Figure 2A), and in the cell lines derived from primary patient xenografts (Figure 2B). Chloroquine consistently resulted in less caspase- 3 activation than ABT-737, and in some lines - including both lines derived from primary patient xenografts - led to almost no caspase-3 activation as a single agent, yet substantially increased activated caspase-3 when combined with ABT-737.

Upon activation of autophagy, the LC3-I protein is phosphatidylethanolamine (PE) conjugated to form LC3-II and is preferentially translocated to the membranes of autophagosomes. The appearance of an LC3-II band on western blots is a generally accepted indicator of autophagy [32]. If apoptotic induction by ABT-737 leads to a protective autophagic response in cancer cells, then we might expect to see detectable markers of upregulated autophagy following ABT-737 exposure, particularly in the context of decreased flux through the autophagic 

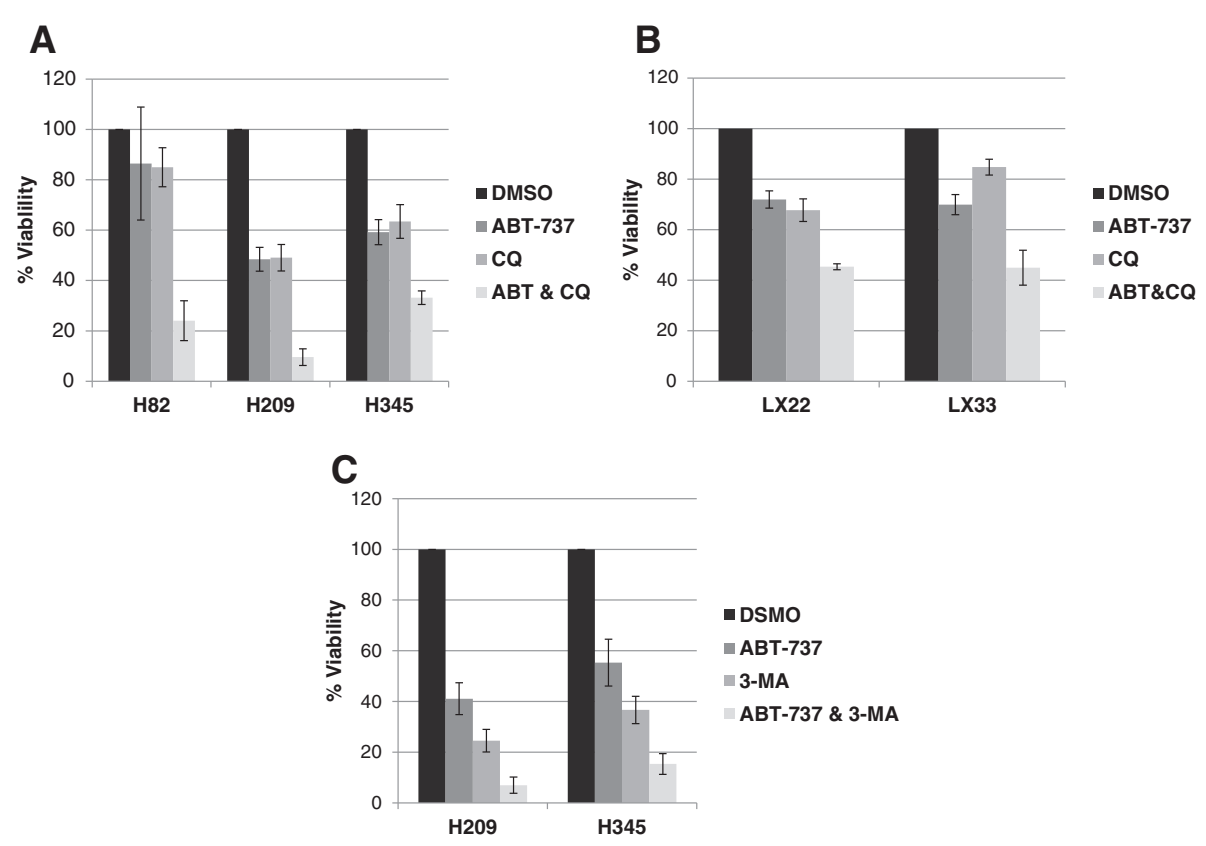

Figure 1 Combination treatment of ABT-737 with autophagy inhibitors results in decreased proliferation in SCLC cell lines. Cells were plated in 96-well plates and treated with DMSO, ABT-737, chloroquine (CQ), and the combination of ABT-737 and chloroquine. At 72 hours posttreatment they were assayed using the MTS cell proliferation assay. Commercially available cell lines are shown in $\mathbf{A}$ and cell lines derived from low passage cultures of primary patient xenografts in B. Doses chosen were based on initial determination of $\mathrm{IC}_{50}$ values and were as follows:

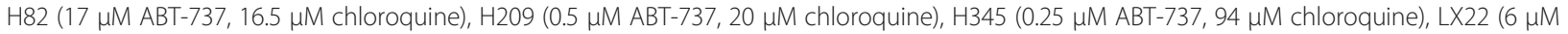
ABT-737, $40 \mu \mathrm{M}$ chloroquine), and LX33 (0.8 $\mu \mathrm{M}$ ABT-737, $33 \mu \mathrm{M}$ chloroquine). C. Similar treatments were performed combining ABT-737 and 3methyladenine, another autophagy inhibitor. 3-methyladenine doses were $5 \mathrm{mM}$ for both lines. Error bars: standard deviation (SD); $\mathrm{n}=4$.

pathway by concomitant treatment with chloroquine. To test this idea, we performed similar single agent and combination drug treatments, and isolated protein to blot for levels of LC3-II. No LC3-II was detectable in vehicle control-treated cells, or in cells treated with single agent ABT-737, but was clearly evident in all cell lines when treated with chloroquine, and further enhanced by the combination of chloroquine and ABT-737 (Figure 3A). Measurement of LC3-II levels showed that for each cell line, there was an increase in LC3-II in the combination treatment compared to chloroquine alone (Figure 3B). These data suggest that ABT-737 may in fact trigger autophagy, as previously suggested [33], but that flux through the pathway precludes detection of LC3-II unless an inhibitor of completion of autophagic digestion such as chloroquine is present. To more conclusively assess whether ABT-737 induces autophagy, we examined ABT737 treated cell lines by transmission electron microscopy (TEM) to look for the presence of characteristic autophagosomes. Representative images shown in Figure 3C
A

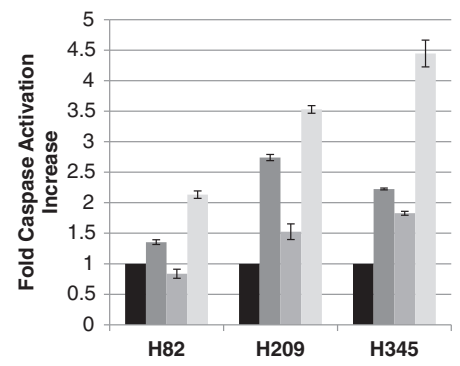

B

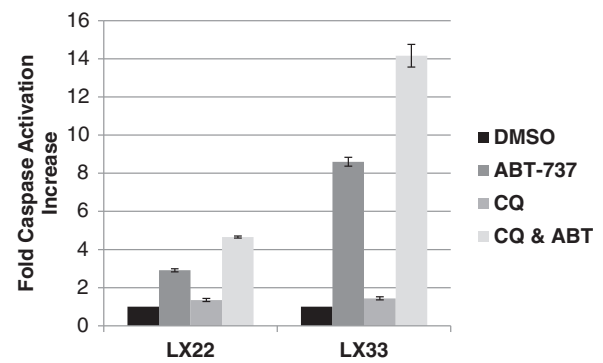

Figure 2 Combination of ABT-737 and chloroquine results in increased induction of apoptosis in SCLC cell lines. Cells were treated at the doses listed in Figure 1 for ABT-737 and chloroquine or the combination for 72 hrs, and then assayed using the Caspase-Glo 3/7 assay. Luminescence is directly proportional to the amount of caspase-3 activation. Commercially available cell lines are shown in $\mathbf{A}$ and cell lines derived from patient xenografts are shown in $\mathbf{B}$. Error bars: SD; $\mathrm{n}=4$. 

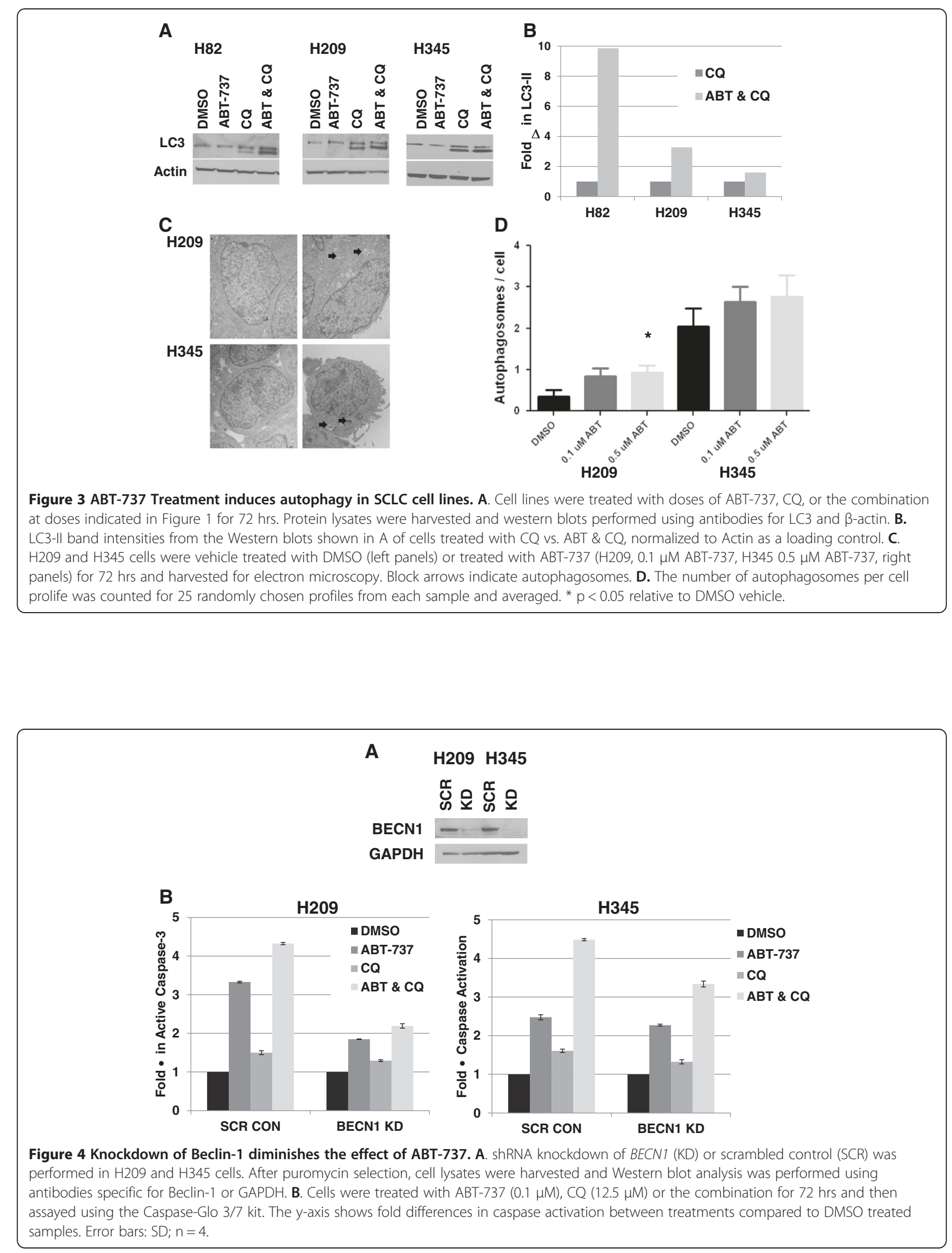
and quantitative data presented in Figure 3D confirm a small dose-dependent increase in autophagosome formation in response to ABT-737 in both H209 and H345 cells (Figure 3C and D). However, statistical significance was only reached in $\mathrm{H} 209$ when comparing $0.5 \mu \mathrm{M}$ ABT-737 treatment to control $(\mathrm{p}=0.025)$.

While both chloroquine and 3MA appeared to enhance the efficacy of ABT-737 against SCLC, these pharmacologic agents could both be influencing cell proliferation and apoptosis via pathways other than autophagy. In addition, chloroquine may affect completion of autophagy in cells committed to autophagic induction, rather than influencing initiation of this pathway. To further dissect the potential interactions between the apoptosis and autophagy pathways in SCLC, we adopted a genetic approach, using shRNA knockdown of BECN1 in both H209 and H345 cells (Figure 4A). Beclin-1 is of particular interest, both an essential autophagy protein that controls the first steps of autophagic commitment, and as a known binding partner of the primary targets of ABT-737, Bcl-2 and Bcl- $\mathrm{x}_{\mathrm{L}}$. Surprisingly, and in sharp contrast to the results obtained with chloroquine and 3MA, knockdown of BECN1 decreased caspase-3 activation in response to ABT-737 and the combination of ABT-737 and chloroquine (Figure 4B).

Similar suppressive effects on apoptotic induction were seen in H209 and H345. Taken together with data demonstrating that ABT-737 can activate autophagy (as determined by LC3-II levels and autophagosome formation), and that two inhibitors of autophagy (chloroquine and 3MA) can augment ABT-737 efficacy, the entirely unexpected results obtained using BECN1 knockdown suggest that the complex of Bcl-2 and Beclin-1 may have functional significance in the regulation of apoptosis by Bcl-2, independent of the suppressive effect on autophagy.

It is possible that the inhibitors of autophagy used here could have biological effects on the regulation of cell survival independent of the autophagy pathway. Notably, resistance to ABT-737 in SCLC cell lines has been associated with increased expression of the anti-apoptotic Bcl-2 family member Mcl-1, which is not effectively inhibited by ABT-737, and decreased expression of NOXA, a proapoptotic Bcl-2 family member which inhibits Mcl-1 [34]. What is more, both chloroquine and 3-MA have been shown to decrease Mcl-1 expression in neutrophils [35]. Together, these observations suggest an alternative and potentially complementary mechanism by which the combination of ABT-737 and chloroquine could effectively inhibit SCLC growth. To explore whether these mechanisms might be operant in our cell lines, we performed Western blotting of Mcl-1, NOXA, and other relevant Bcl-2 family members in H82, H209, and H345 cells treated with ABT-737, chloroquine, or combinations of both agents. As seen in Figure 5, in at least two of these lines (H209 and H345), combination therapy was associated with marked decrease in Mcl-1. H209 did not express detectable NOXA under any conditions, but in the other two lines, combination therapy was also associated with NOXA upregulation. These data suggest that at least part of the combinatorial effect of ABT-737 and chloroquine may be mediated by alteration in the relative balance of pro- and anti-apoptotic Bcl-2 family members, promoting cell death.

Finally, given the observed enhanced cytotoxicity seen in vitro through combination of ABT-737 and chloroquine, we sought to explore whether this combination could improve tumor control in vivo. Cohorts of established tumor xenografts of the cell line H209, as well as three SCLC PDX models, LX22, LX33, and LX44, were treated with daily intraperitoneal injection of chloroquine, ABT-737, combination, or vehicle-treated controls, and tumor volume was monitored over time. The combination was superior to single agent treatment in the $\mathrm{H} 209$ xenografts $(\mathrm{p}=0.005$ for comparison with ABT-737 alone), but showed no evidence of superiority over ABT-737 alone in the treatment of the primary xenograft models ( $p>0.5$ for comparison with ABT-737 alone in the primary xenografts) (Figure 6). The interaction p-value remains significant for H209 with a Bonferroni correction for multiple testing $(\mathrm{p}=0.02)$.

\section{Discussion}

In this study we sought to assess whether the efficacy of the targeted Bcl-2 inhibitor, ABT-737, against SCLC could be improved by concurrent inhibition of autophagy with the clinically available agent chloroquine. In vitro analyses of the combination of ABT-737 and chloroquine in several SCLC cell lines did show a decrease in viability and a concomitant increase in caspase- 3 activation. In addition, we found evidence that ABT-737 induced autophagy in several cell lines by the conversion of LC3-I to LC3-II seen on western blots and increased autophagosome formation as documented by TEM.

However, a targeted genetic approach to blocking entry into autophagy based on shRNA knockdown of BECN1 yielded the opposite result: a consistent decrease in apoptotic response to ABT-737, with or without chloroquine. There are several possible explanations for these seemingly contradictory results. First, the effects seen with chloroquine may not have been specific to the autophagy pathway. Indeed, we were able to demonstrate that the combination of ABT-737 and chloroquine can lead to marked inhibition of Mcl-1 and upregulation of NOXA, both of which would serve to facilitate apoptotic induction, independent of an effect on autophagy. It is known that chloroquine can also act on several other pathways including those involved in immune modulation and DNA damage [17].

Second, while chloroquine inhibits the late stages of autophagy, i.e. the fusion of autophagosomes with lysosomes 


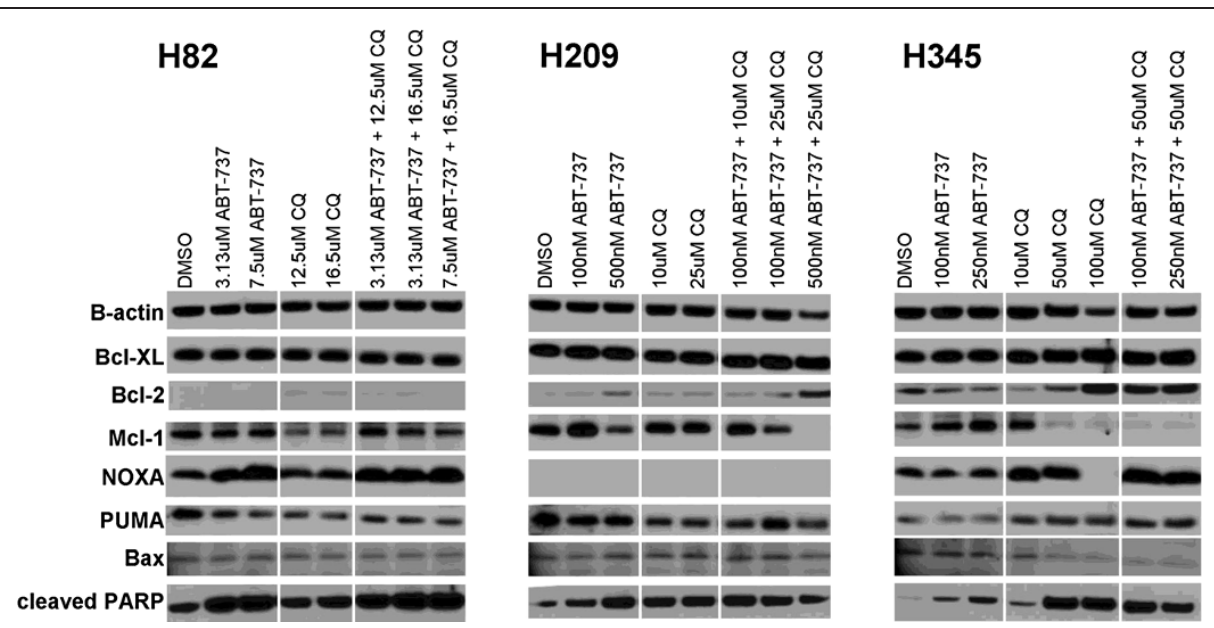

Figure 5 ABT-737 and chloroquine combination therapy alters the expression levels of apoptotic regulatory factors in SCLC. The cell lines H82, H209 and H345 were treated with DMSO (control), ABT-737, CQ, and combinations of the two drugs for 72 hours before protein lysates were isolated. Doses of ABT-737 and CQ that were below or approximating the cell line specific $I_{50}$ values for each agent were chosen. Betaactin is shown as an internal loading control and cleaved-PARP is shown for relative induction of apoptosis. NOXA was undetectable in H209 and $\mathrm{BCl}-2$ was barely detectable in $\mathrm{H} 82$.

leading to degradation of vesicular contents, Beclin-1 is required for an initial commitment toward autophagy. It is possible that successful initiation of autophagy without the ability to complete the process leads to intracellular accumulation of toxic materials, leading to enhanced apoptotic cell death, while blockade of entry into this pathway does not. Some reports do suggest that specific stage of autophagy inhibition can result in different therapeutic outcomes $[5,36]$. Our data with 3MA would argue against this explanation to some extent: 3MA inhibits initiation of autophagy at a stage closely related to that of Beclin-1, but unlike Beclin-1 suppression, augmented the apoptotic response to ABT-737 in multiple lines. 3MA is a class I and class III PI3K inhibitor [14], and could, like
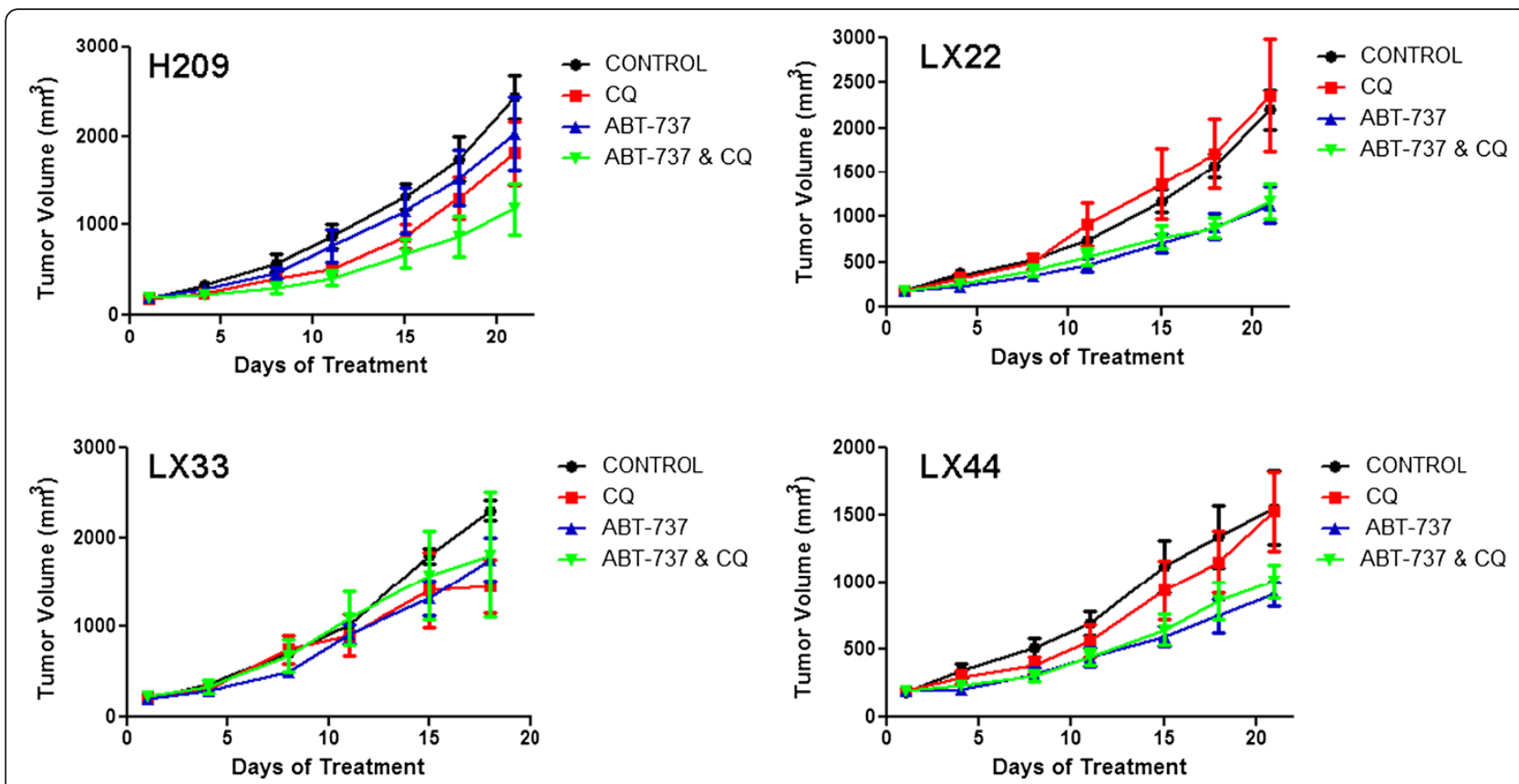

Figure 6 Combination of ABT-737 and chloroquine does not result in increased efficacy in xenografts models. Tumor bearing Nu/Nu mice (4-7 per treatment arm) were treated daily with ABT-737 (100 mg/kg, IP),CQ (60 mg/kg, IP), combination, or an equal amount of vehicle control for 18-21 days. Tumor volumes were measured every three days. Group means and standard error of the mean for each measurement are shown. 
chloroquine, have other relevant effects leading to enhanced programmed cell death.

Third, and perhaps most intriguingly, Beclin-1 and $\mathrm{Bcl}-2$ are known to directly interact, and shRNA suppression of BECN1 may affect Bcl-2 function or localization independent of any effect on induction of autophagy. While previous data suggest that Beclin-1 does not affect the anti-apoptotic activity or localization of Bcl-2, this finding may be context and cell type-specific; these studies were performed in HeLa cervical carcinoma cells and mouse embryonic fibroblasts [37]. One possible model to explain our results would be if Beclin-1 sequesters some fraction of the cellular Bcl-2 away from the mitochondrial membrane, where $\mathrm{Bcl}-2$ primarily acts to suppress outer mitochondrial membrane disruption. Targeted loss of Beclin-1 could lead to a preferential localization of $\mathrm{Bcl}-2$ to mitochondria, decreasing ABT-737 efficacy by functional upregulation of the target. The novel observations in this study suggest several testable hypotheses to be addressed in future studies, which may provide additional insights into the interface between regulators of apoptosis and autophagy in SCLC.

\section{Conclusions}

Several ongoing clinical trials seek to evaluate the efficacy of autophagy inhibitors in combination with cytotoxic therapies in cancer types including SCLC. The data presented here have important implications for studies in SCLC. Different strategies for modulating autophagy can have markedly discordant effects on the efficacy of cytotoxic therapies. It will be important to study the modulation of autophagy closely in relevant preclinical models to anticipate possible combinatorial effects.

In work presented here, we analyzed for the first time the effect of combining ABT-737 and chloroquine in SCLC models, including assessment of activity against PDX tumors, models that may better reflect the biology of disease than do cell line-based xenografts [31,38]. Our in vivo xenograft data, particularly in the three primary xenograft models, suggest that despite some intriguing molecular interactions, the combination of ABT-737 and chloroquine is unlikely to have significant anti-cancer activity against SCLC, relative to ABT-737 alone.

\section{Methods}

\section{Cell lines}

All cell lines were obtained from American Type Culture Collection and grown in the recommended media. Primary xenograft cell lines (LX) were derived from patient tumors as previously described [31].

\section{MTS cell proliferation assays}

Cells were plated in quadruplicate and treated with ABT-737 and chloroquine for 72 hours at indicated doses.
MTS cell proliferation assays were performed following manufacturer instructions using the CellTiter 96 AQueous One Solution Cell Proliferation Assay (Promega).

Caspase-Glo 3/7 assay. Assay was performed following manufacturer instructions (Promega). Briefly, 20,000 viable cells from each sample were transferred to 96-well white-walled plates in 75-100 $\mu \mathrm{l}$ total volume of culture medium. An equal volume of Caspase-Glo 3/7 Reagent was added to each well. Assays were performed in quadruplicate. Plates were covered and incubated at room temperature for 1-2 hours before measuring luminescence on a plate reading luminometer. The luminescence reading is directly proportional to caspase-3 activity.

\section{Western blotting}

Protein was isolated from cell pellets using radioimmunoprecipitation assay buffer (Sigma) and run on 10 or $12 \%$ Bis-Tris gels with MOPS or MES running buffer (Invitrogen). Membranes were probed with antibodies for LC3, Beclin-1, GAPDH, $\beta$-Actin (Santa Cruz), Mcl-1 (BD Pharmigen; \#559027), Bax (Santa Cruz; \#sc-6236), Bcl-XL (Cell Signaling; \#2764), Bcl-2 (Santa Cruz; \#sc-492), NOXA (Imgenex; \#IMG-349A), PUMA (Cell Signaling; \#4976), and cleaved PARP (Cell Signaling; \#5625) following manufacturer instructions.

\section{Electron microscopy}

Samples were fixed in $2.5 \%$ glutaraldehyde, $3 \mathrm{mM} \mathrm{CaCl}_{2}$, $1 \%$ sucrose, in $0.1 \mathrm{M}$ sodium cacodylate buffer, $\mathrm{pH} 7.2$ for one hour at room temperature. After buffer rinse, samples were postfixed in $1 \%$ osmium tetroxide in buffer $(1 \mathrm{hr})$ on ice in the dark. Following a $\mathrm{DH}_{2} \mathrm{O}$ rinse, plates were stained with $2 \%$ aqueous uranyl acetate $(0.22 \mu \mathrm{m}$ filtered, $1 \mathrm{hr}$, dark), dehydrated in a graded series of ethanol and embedded in Eponate 12 (Ted Pella) resin. Samples were polymerized at $60^{\circ} \mathrm{C}$ overnight. Thin sections, 60 to $90 \mathrm{~nm}$, were cut with a diamond knife on the ReichertJung Ultracut E ultramicrotome and picked up with naked 200 mesh copper grids. Grids were stained with $2 \%$ uranyl acetate in 50\% methanol and observed with a Philips CM 120 at $80 \mathrm{kV}$. Images were captured with an Advanced Microscopy Techniques transmission electron microscopy $(1 \mathrm{~K} \times 1 \mathrm{~K})$ camera.

\section{Lentiviral shRNA and CDNA overexpression experiments}

Lentiviral particles were generated using a three-plasmid system and infected as per the RNAi Consortium Library Production and Performance Protocols, Broad Institute [39]. shRNA constructs were obtained from the Broad RNAi Consortium and screened for knockdown. The BECN1 shRNA clone ID TRCN0000033550 was used these experiments. pLKO.1-shRNA scramble vector was obtained from Dr. David M. Sabatini through Addgene (Addgene plasmid 1864) as previously described [40]. 


\section{In vivo studies}

Patient-derived xenograft tumor models LX22, LX33, and LX44 were generated as previously described [24,31]. NCIH209 cells $\left(1 \times 10^{7}\right)$, or SCLC patient derived tumor cells LX22 $\left(1.5 \times 10^{6}\right)$, LX33 $\left(1.6 \times 10^{6}\right)$, and LX44 $\left(3.5 \times 10^{6}\right)$, were suspended in a 1:1 ratio of PBS and matrigel (BD Biosciences). Cell suspensions were injected subcutaneously on right hind flanks of female nu/nu $6-8$ week old mice (Charles River). Drug treatments began when tumor sizes reached $200 \mathrm{~mm}^{3}$. Mice were treated daily with $100 \mathrm{mg} / \mathrm{kg}$ ABT-737 (IP), $60 \mathrm{mg} / \mathrm{kg}$ chloroquine (IP), combination or vehicle treated for 21 days. Tumors were measured with a manual caliper every 3 days and volumes were calculated using the formula: tumor weight $(\mathrm{mg})=$ [length $(\mathrm{mm}) \mathrm{x}$ width $\left.{ }^{2}\left(\mathrm{~mm}^{2}\right)\right] / 2$. In order to test whether treatment inhibited tumor growth, we used a mixed effects linear model comparing tumor growth between ABT-737 and ABT-737 and CQ treatment groups, modeling the log of tumor size over time and including an interaction term. All in vivo experiments were conducted with protocol approval by the Johns Hopkins Animal Care and Use Committee.

\section{Statistical analyses}

The student's $t$-test was used to compare combination treatment effects vs. single agent treatment effects in cell lines. For in vivo analyses of whether treatment inhibited tumor growth, we used a mixed effects linear model comparing tumor growth between treatment groups, modeling the log of tumor size over time and including an interaction term. A $\mathrm{p}$ value $<0.05$ was considered significant.

\section{Abbreviations \\ 3MA: 3-Methyladenine; CQ: Chloroquine; MTS: (3-(4,5-dimethylthiazol-2-yl)-5- (3-carboxymethoxyphenyl)-2-(4-sulfophenyl)-2H-tetrazolium); PDX: Patient- derived xenograft; SCLC: Small cell lung cancer; TEM: Transmission electron microscopy.}

\section{Competing interests}

The authors have no competing interests with regard to the data presented here.

\section{Authors' contributions}

RLZ conducted the majority of experiments reported here, and drafted the manuscript. EEG conducted additional essential in vitro experiments. ID assisted with all in vivo experiments. SM assisted with molecular and cell culture studies. LM provided statistical support. CLH provided key intellectual input, practical advice on experimental approaches, and assisted with data interpretation. CMR conceived of the study, participated in its design and coordination, and assisted with data interpretation and manuscript writing. All authors read and approved the final manuscript.

\section{Acknowledgements}

The authors would like to thank members of the Rudin and Hann laboratories for discussion and advice regarding this work. In addition, we would like to thank Barbara Smith for her technical assistance with electron microscopy. Grant support was provided by the Flight Attendant Medical Research Institute, the Burroughs Wellcome Fund, the Anti-Cancer Drug Development Training Grant (NIH T32 CA009243), and the Cancer Center Support Grant (NCI P30 CA006973).
Received: 27 August 2012 Accepted: 27 February 2013

Published: 2 March 2013

\section{References}

1. Kundu M, Thompson CB: Autophagy: basic principles and relevance to disease. Annu Rev Pathol 2008, 3:427-455.

2. White E, DiPaola RS: The double-edged sword of autophagy modulation in cancer. Clin Cancer Res 2009, 15:5308-5316.

3. Bursch W, Ellinger A, Kienzl H, Torok L, Pandey S, Sikorska M, Walker R, Hermann RS: Active cell death induced by the anti-estrogens tamoxifen and ICI 164384 in human mammary carcinoma cells (MCF-7) in culture: the role of autophagy. Carcinogenesis 1996, 17:1595-1607.

4. Ertmer A, Huber V, Gilch S, Yoshimori T, Erfle V, Duyster J, Elsasser HP, Schatzl HM: The anticancer drug imatinib induces cellular autophagy. Leukemia 2007, 21:936-942.

5. Kanzawa T, Germano IM, Komata T, Ito H, Kondo Y, Kondo S: Role of autophagy in temozolomide-induced cytotoxicity for malignant glioma cells. Cell Death Differ 2004, 11:448-457.

6. Paglin S, Hollister T, Delohery T, Hackett N, McMahill M, Sphicas E, Domingo $D$, Yahalom J: A novel response of cancer cells to radiation involves autophagy and formation of acidic vesicles. Cancer Res 2001, 61:439-444

7. Yang C, Kaushal V, Shah SV, Kaushal GP: Autophagy is associated with apoptosis in cisplatin injury to renal tubular epithelial cells. Am J Physiol Renal Physiol 2008, 294:F777-F787.

8. Amaravadi RK, Thompson CB: The roles of therapy-induced autophagy and necrosis in cancer treatment. Clin Cancer Res 2007, 13:7271-7279.

9. Amaravadi RK, Lippincott-Schwartz J, Yin XM, Weiss WA, Takebe N, Timmer W, DiPaola RS, Lotze MT, White E: Principles and current strategies for targeting autophagy for cancer treatment. Clin Cancer Res 2011, 17:654-666.

10. Abedin MJ, Wang D, McDonnell MA, Lehmann U, Kelekar A: Autophagy delays apoptotic death in breast cancer cells following DNA damage. Cell Death Differ 2007, 14:500-510.

11. Amaravadi RK, Yu D, Lum JJ, Bui T, Christophorou MA, Evan Gl, ThomasTikhonenko A, Thompson CB: Autophagy inhibition enhances therapyinduced apoptosis in a Myc-induced model of lymphoma. J Clin Invest 2007, 117:326-336.

12. Guo XL, Li D, Hu F, Song JR, Zhang SS, Deng WJ, Sun K, Zhao QD, Xie XQ, Song YJ, et al: Targeting autophagy potentiates chemotherapy-induced apoptosis and proliferation inhibition in hepatocarcinoma cells. Cancer Lett 2012, 320:171-179.

13. Li J, Hou N, Faried A, Tsutsumi S, Takeuchi T, Kuwano H: Inhibition of autophagy by 3-MA enhances the effect of 5-FU-induced apoptosis in colon cancer cells. Ann Surg Oncol 2009, 16:761-771.

14. Maycotte $P$, Thorburn A: Autophagy and cancer therapy. Cancer Biol Ther 2011, 11:127-137.

15. Kondo $Y$, Kanzawa $T$, Sawaya $R$, Kondo $S$ : The role of autophagy in cancer development and response to therapy. Nat Rev Cancer 2005, 5:726-734.

16. Solomon VR, Lee H: Chloroquine and its analogs: a new promise of an old drug for effective and safe cancer therapies. Eur J Pharmacol 2009, 625:220-233.

17. Chen N, Karantza V: Autophagy as a therapeutic target in cancer. Cancer Biol Ther 2011, 11:157-168.

18. Siegel R, Naishadham D, Jemal A: Cancer statistics, 2012. CA Cancer J Clin 2012, 62:10-29.

19. Jackman DM, Johnson BE: Small-cell lung cancer. Lancet 2005, 366:1385-1396.

20. Ikegaki N, Katsumata M, Minna J, Tsujimoto Y: Expression of bcl-2 in small cell lung carcinoma cells. Cancer Res 1994, 54:6-8.

21. Stefanaki K, Rontogiannis D, Vamvouka C, Bolioti S, Chaniotis V, Sotsiou F, Vlychou M, Delidis G, Kakolyris S, Georgoulias V, Kanavaros P: Immunohistochemical detection of bcl2, p53, mdm2 and p21/waf1 proteins in small-cell lung carcinomas. Anticancer Res 1998, 18:1167-1173.

22. Zangemeister-Wittke U, Schenker T, Luedke GH, Stahel RA: Synergistic cytotoxicity of bcl-2 antisense oligodeoxynucleotides and etoposide, doxorubicin and cisplatin on small-cell lung cancer cell lines. $\mathrm{Br} J$ Cancer 1998, 78:1035-1042.

23. Oltersdorf T, Elmore SW, Shoemaker AR, Armstrong RC, Augeri DJ, Belli BA, Bruncko M, Deckwerth TL, Dinges J, Hajduk PJ, et al: An inhibitor of Bcl-2 family proteins induces regression of solid tumours. Nature 2005, 435:677-681.

24. Hann CL, Daniel VC, Sugar EA, Dobromilskaya I, Murphy SC, Cope L, Lin X, Hierman JS, Wilburn DL, Watkins DN, Rudin CM: Therapeutic efficacy of 
ABT-737, a selective inhibitor of BCL-2, in small cell lung cancer. Cancer Res 2008, 68:2321-2328.

25. Gandhi L, Camidge DR, Ribeiro de Oliveira M, Bonomi P, Gandara D, Khaira D, Hann CL, McKeegan EM, Litvinovich E, Hemken PM, et al: Phase I study of navitoclax (ABT-263), a novel Bcl-2 family inhibitor, in patients with smallcell lung cancer and other solid tumors. J Clin Oncol 2011, 29:909-916.

26. Rudin CM, Hann CL, Garon EB, Ribeiro de Oliveira M, Bonomi PD, Camidge DR, Chu Q, Giaccone G, Khaira D, Ramalingam SS, et al: Phase II study of single-agent navitoclax (ABT-263) and biomarker correlates in patients with relapsed small cell lung cancer. Clin Cancer Res 2012, 18:3163-3169.

27. Pattingre S, Tassa A, Qu X, Garuti R, Liang XH, Mizushima N, Packer M, Schneider MD, Levine B: Bcl-2 antiapoptotic proteins inhibit beclin 1dependent autophagy. Cell 2005, 122:927-939.

28. Maiuri MC, Criollo A, Tasdemir E, Vicencio JM, Tajeddine N, Hickman JA, Geneste $\mathrm{O}$, Kroemer G: BH3-Only proteins and BH3 mimetics induce autophagy by competitively disrupting the interaction between beclin 1 and BCl-2/BCl-X(L). Autophagy 2007, 3:374-376.

29. Maiuri MC, Le Toumelin G, Criollo A, Rain JC, Gautier F, Juin P, Tasdemir E, Pierron G, Troulinaki K, Tavernarakis N, et al: Functional and physical interaction between $\mathrm{BCl}-\mathrm{X}(\mathrm{L})$ and a $\mathrm{BH} 3$-like domain in beclin-1. EMBO $\mathrm{J}$ 2007, 26:2527-2539.

30. Gao P, Bauvy C, Souquere S, Tonelli G, Liu L, Zhu Y, Qiao Z, Bakula D, Proikas-Cezanne T, Pierron G, et al: The Bcl-2 homology domain 3 mimetic gossypol induces both beclin 1-dependent and beclin 1-independent cytoprotective autophagy in cancer cells. J Biol Chem 2010, 285:25570-25581.

31. Daniel VC, Marchionni L, Hierman JS, Rhodes JT, Devereux WL, Rudin CM, Yung R, Parmigiani $G$, Dorsch $M$, Peacock CD, Watkins DN: A primary xenograft model of small-cell lung cancer reveals irreversible changes in gene expression imposed by culture in vitro. Cancer Res 2009, 69:3364-3373.

32. Klionsky DJ, Abdalla FC, Abeliovich $\mathrm{H}$, Abraham RT, Acevedo-Arozena A, Adeli K, Agholme L, Agnello M, Agostinis P, Aguirre-Ghiso JA, et al: Guidelines for the use and interpretation of assays for monitoring autophagy. Autophagy 2012, 8:445-544

33. Malik SA, Orhon I, Morselli E, Criollo A, Shen S, Marino G, BenYounes A, Benit P, Rustin P, Maiuri MC, Kroemer G: BH3 Mimetics activate multiple pro-autophagic pathways. Oncogene 2011, 30:3918-3929.

34. Hauck P, Chao BH, Litz J, Krystal GW: Alterations in the noxa/Mcl-1 axis determine sensitivity of small cell lung cancer to the BH3 mimetic ABT737. Mol Cancer Ther 2009, 8:883-892.

35. Pliyev BK, Menshikov M: Differential effects of the autophagy inhibitors 3methyladenine and chloroquine on spontaneous and TNF-alpha-induced neutrophil apoptosis. Apoptosis 2012, 17:1050-1065.

36. Shingu T, Fujiwara K, Bogler O, Akiyama Y, Moritake K, Shinojima N, Tamada $Y$, Yokoyama T, Kondo S: Inhibition of autophagy at a late stage enhances imatinib-induced cytotoxicity in human malignant glioma cells. Int J Cancer 2009, 124:1060-1071.

37. Ciechomska IA, Goemans GC, Skepper JN, Tolkovsky AM: BCl-2 complexed with beclin-1 maintains full anti-apoptotic function. Oncogene 2009, 28:2128-2141.

38. Tentler JJ, Tan AC, Weekes CD, Jimeno A, Leong S, Pitts TM, Arcaroli JJ, Messersmith WA, Eckhardt SG: Patient-derived tumour xenografts as models for oncology drug development. Nat Rev Clin Oncol 2012, 9:338-350.

39. Moffat J, Grueneberg DA, Yang X, Kim SY, Kloepfer AM, Hinkle G, Piqani B, Eisenhaure TM, Luo B, Grenier JK, et al: A lentiviral RNAi library for human and mouse genes applied to an arrayed viral high-content screen. Cell 2006, 124:1283-1298.

40. Sarbassov DD, Guertin DA, Ali SM, Sabatini DM: Phosphorylation and regulation of Akt/PKB by the rictor-mTOR complex. Science 2005, 307:1098-1101.

doi:10.1186/1476-4598-12-16

Cite this article as: Zinn et al.: Combination treatment with ABT-737 and chloroquine in preclinical models of small cell lung cancer. Molecular Cancer 2013 12:16.

\section{Submit your next manuscript to BioMed Central and take full advantage of:}

- Convenient online submission

- Thorough peer review

- No space constraints or color figure charges

- Immediate publication on acceptance

- Inclusion in PubMed, CAS, Scopus and Google Scholar

- Research which is freely available for redistribution

Submit your manuscript at www.biomedcentral.com/submit
C Biomed Central 